$\xi=-1$

\title{
Influence of Variable Viscosity on Peristaltic Motion of a Viscoelastic Fluid in a Tapered Microfluidic Vessel
}

\author{
J. Prakash ${ }^{1}{ }^{*}$, E.P.Siva $^{2}$, A.Govindarajan ${ }^{3}$ and M.Vidhya ${ }^{4}$ \\ ${ }^{1}$ Department of Mathematics, Srinivasa Ramanujan Center, SASTRA Deemed University, Kumbakonam - 612001, Tamilnadu, India \\ ${ }^{2,3}$ Department of Mathematics, SRM Institute of Science and Technology, Kattankulathur- 603203, Tamilnadu, India \\ ${ }^{4}$ Department of Mathematics, Sathiyabama Institute of Science and Technology, Sholinganallur -600119 Tamilnadu, India \\ *Corresponding author E-mail: prakashjayavel@yahoo.co.in
}

\begin{abstract}
The peristaltic flow of a viscoelastic fluid in the tapered microchannel with variable viscosity is investigated. This study is reinvigorated by discovering fluid dynamic in peristaltic motion as signified by biological flows, pharmacodynamics and gastro-intestinal motility enhancement. The microchannel non-uniform and asymmetry is developed by choosing a peristaltic wave train on the wall with different amplitudes and phases. The flow analysis has been arisen for low Reynolds number and long wavelength case. The solutions for stream function, axial velocity and pressure gradient are obtained. The effects of pertinent parameters on the average pressure rise per wavelength are investigated by means of numerical integration. The axial velocity and phenomena of trapping are further discussed.
\end{abstract}

Keywords: Peristaltic transport; Variable viscosity; viscoelastic fluid; Tapered microchannel.

\section{Introduction}

In recent years, the investigation of peristaltic transport in a channel is well knows as a crucial type of flow occurring in numerous practical applications, like urine transport from kidney to bladder through the ureter, roller and finger pumps, chyme motion in the gastrointestinal tract, movement of ovum in the fallopian tube, transport of lymph in the lymphatic vessels and vasomotion of small blood vessels such as arterioles, powder technology and many others [1-11]. The minority of the physiological systems in human body cannot be formed by a symmetrical channel, particularly the sagittal cross section of the uterus. Eytan and Elad [12] and Eytan et al. [13] developed the intra uterine fluid in the sagittal cross section of the uterus by an asymmetric channel in lubrication approach. Mishra and Ramachandra Rao [14] extended the flow in an asymmetric channel generated by peristaltic waves propagating on the walls. Hayat and Ali [15] studied the peristaltic motion of Carreau fluid in an asymmetric channel.

Vajravelu et al., have developed a peristaltic transport of a Williamson fluid in asymmetric channels with permeable walls [16] A good number of the studies in the literature have been carried in uniform geometry only but it is well known that in most of the practical applications, such as the physiological body organs, peristaltic mechanism involved in small blood vessels, lymphatic vessel, intestine, ducts afferents of the male reproductive tracts and in transport of spermatozoa in the cervical canal are generally observed to be non-uniform flow geometry [17-20]. Srivastava and Srivastava [21] observed the peristaltic flow in the vas deferens by assuming it to be a non-uniform diverging channel and a tube. They appeared at a more realistic representation by expressing non-Newtonian (Power law fluid) fluid flow in a non-uniform tube.All the above-citied studies point out that physiological fluid with constant viscosity but its fails to give better understanding when peristaltic mechanism involved in small blood vessels, lym- phatic vessel, intestine, ductus efferentes of the male reproductive tracts and in transport of spermatozoa in the cervical canal. Therefore, it is extremely attractive to include the effect of variable viscosity instead of making an allowance for the viscosity of the fluid to be constant. Hayat and Ali [22] considered the peristaltic transport of a Newtonian fluid with variable viscosity in an asymmetric channel. The influence of slip condition on the peristaltic motion of a magnetohydrodynamic (MHD) viscous fluid with variable viscosity has been reported by Ali et al. [23].

Motivated by these studied that the general case of asymmetric wall oscillation in non-uniform channel may also exist in biological conduits, e.g., the uterus [24]. In this learn, the present study is an asymmetric flow of a particulate suspension in a non-uniform microchannel induced by sinusoidal peristaltic waves. In addition that, the most generalized form of a two dimensional channel is suggested to study the peristalsis, so called as the tapered microchannel or generalized micro-tapered asymmetric channel. To the best of our knowledge, no investigation has been made yet to analyze the effect variable viscosity on the peristaltic flow of a viscoelastic fluid in the tapered microfluidic vessel. The governing equations are modeled and then solved analytically by using long wavelength and low-Reynolds number assumptions. Expressions for the axial velocity, stream function and average pressure rise per wavelength are presented. Numerical computation has also been performed for the average pressure rise. The effects are presented and analyzed for an adequate range of influential physical parameters.

\section{Mathematical Formulation}

We consider a two - dimensional flow of a viscoelastic fluid with variable viscosity through an asymmetric microchannel of nonuniform cross-section with sinusoidal wave trains propagate on the channel walls with unvarying speed c. Let $Y=H_{1}$ and $Y=H_{2}$ be 
respectively the upper and lower wall boundaries of the tapered microchannel of width $2 d$. The geometry of the proposed wall surfaces are defined (Fig.1) as

$$
\begin{aligned}
& \bar{H}_{2}\left(\bar{X}, t^{\prime}\right)=d+m^{\prime} \bar{X}+a_{2} \cos ^{2}\left[\frac{\pi}{\lambda}\left(\bar{X}-c t^{\prime}\right)\right] \\
& \bar{H}_{1}\left(\bar{X}, t^{\prime}\right)=-d-m^{\prime} \bar{X}-a_{1} \cos ^{2}\left[\frac{\pi}{\lambda}\left(\bar{X}-c t^{\prime}\right)+\phi\right] .
\end{aligned}
$$

where $d$ is the half-width of the channel, $a_{1}$ and $a_{2}$ are the amplitudes of lower and upper wall respectively, $c$ is the phase speed of the wave, $k^{\prime}(<<1)$ is the non - uniform parameter, $\lambda$ is the wave length and the phase difference $\phi$ varies in the range $0 \leq \phi \leq \pi, \phi=0$ corresponds to symmetric channel with waves out of phase i.e. both walls move towards outward or inward simultaneously.

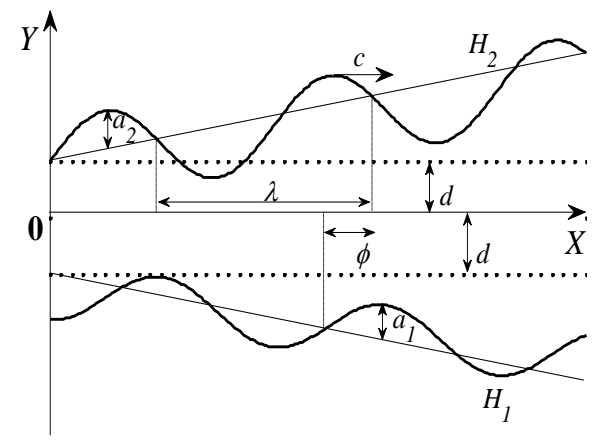

Fig. 1: Schematic diagram of tapered asymmetric channel

The governing equations of motion for the present investigation are

$\frac{\partial \bar{U}}{\partial \bar{X}}+\frac{\partial \bar{V}}{\partial \bar{Y}}=0$

$\rho\left[\frac{\partial \bar{U}}{\partial \bar{t}}+\bar{U} \frac{\partial \bar{U}}{\partial \bar{X}}+\bar{V} \frac{\partial \bar{U}}{\partial \bar{Y}}\right]=-\frac{\partial \bar{P}}{\partial \bar{X}}+2 \frac{\partial}{\partial \bar{X}}\left(\bar{\mu}(\bar{y}) \frac{\partial \bar{U}}{\partial \bar{X}}\right)+\frac{\partial}{\partial \bar{Y}}\left(\bar{\mu}(\bar{y})\left(\frac{\partial \bar{V}}{\partial \bar{X}}+\frac{\partial \bar{U}}{\partial \bar{Y}}\right)\right)$

$\rho\left[\frac{\partial \bar{V}}{\partial \bar{t}}+\bar{U} \frac{\partial \bar{V}}{\partial \bar{X}}+\bar{V} \frac{\partial \bar{V}}{\partial \bar{Y}}\right]=-\frac{\partial \bar{P}}{\partial \bar{Y}}+2 \frac{\partial}{\partial \bar{Y}}\left(\bar{\mu}(\bar{y}) \frac{\partial \bar{V}}{\partial \bar{Y}}\right)+\frac{\partial}{\partial \bar{X}}\left(\bar{\mu}(\bar{y})\left(\frac{\partial \bar{V}}{\partial \bar{X}}+\frac{\partial \bar{U}}{\partial \bar{Y}}\right)\right)$,

where $\bar{U}, \bar{V}$ are the components of velocity along $\bar{X}$ and $\bar{Y}$ directions respectively, $\bar{t}$ is the dimensional time, $\rho$ is the density, $\bar{\mu}(\bar{y})$ is the viscosity function and $\bar{P}$ is the pressure.

The no-slip boundary conditions are

$U=0$ at $Y=H_{1}$ and $Y=H_{2}$.

To make the above equations non-dimensional, it is suitable to introduce the following non-dimensional variable and parameters,

$x=\frac{\bar{X}}{\lambda}, y=\frac{\bar{Y}}{d}, t=\frac{c \bar{t}}{\lambda}, u=\frac{\bar{U}}{c}, v=\frac{\bar{V}}{c}, \delta=\frac{d}{\lambda}, h_{1}=\frac{H_{1}}{d}, h_{2}=\frac{H_{2}}{d}$,

$\mu(y)=\frac{\bar{\mu}(\bar{y})}{\mu_{0}}, \quad a=\frac{a_{1}}{d}, \quad b=\frac{a_{2}}{d}, \quad p=\frac{d^{2} \bar{P}}{c \lambda \mu_{0}}, k=\frac{\lambda k^{\prime}}{d}, \quad \operatorname{Re}=\frac{\rho c d}{\mu_{0}}$.

in which $R e$ is the Reynolds number, $a, b$ are amplitudes of the lower and upper walls respectively, $\delta$ is the wave number and $\mu_{0}$ is the constant viscosity.
Invoking Eq.(7) into Eqs. (3) - (5), we have

$$
\begin{aligned}
R \delta\left[\frac{\partial u}{\partial t}+u \frac{\partial u}{\partial x}+v \frac{\partial u}{\partial y}\right]=-\frac{\partial p}{\partial x} & +2 \delta^{2} \frac{\partial}{\partial x}\left(\mu(y) \frac{\partial u}{\partial x}\right)+\frac{\partial}{\partial y}\left(\mu(y)\left(\delta^{2} \frac{\partial v}{\partial x}+\frac{\partial u}{\partial y}\right)\right) \\
R \delta^{3}\left[\frac{\partial v}{\partial t}+u \frac{\partial v}{\partial x}+v \frac{\partial v}{\partial y}\right]= & -\frac{\partial p}{\partial y}+2 \delta^{2} \frac{\partial}{\partial y}\left(\mu(y) \frac{\partial v}{\partial y}\right) \\
& +\delta^{2} \frac{\partial}{\partial x}\left(\mu(y)\left(\delta^{2} \frac{\partial v}{\partial x}+\frac{\partial u}{\partial y}\right)\right)
\end{aligned}
$$

The corresponding boundary conditions

$$
\begin{aligned}
& u=0 \text { at } y=h_{2}=1+m x+b \cos ^{2}(\pi(x-t)), \\
& u=0 \text { at } y=h_{1}=-1-m x-a \cos ^{2}(\pi(x-t)+\phi) .
\end{aligned}
$$

It is important noted that the dimensionless flow rate $F$ is fluctuating exponentially with the relation $F=Q e^{-A t}$, where $A$ and $Q$ are flow constant. The constant $Q$ is responsible for the negative and non-negative flow rate $F<0$ or $F \geq 0$ according as $Q<0$ or $Q \geq 0$. The negative flow rate shows for backward pumping, when the flow is reversed to the direction of the peristaltic motion. However, the positive pumping denotes to the case when volumetric flow rate and pressure rise are both positive. It was observed experimentally by Kikuchi [25] that the flow rate of blood reduced exponentially with time. It was also recommended that this variation of flow rate is independent on the structural details of the microchannel.

In the forthcoming analysis, we will use [22-23]

$\mu(y)=e^{-\alpha y}$.

where $\alpha$ is the viscosity parameter.

\section{Exact Solution}

Adopting the long wavelength and low Reynolds number approximations [14-16], Eqs. (8-9) can be written as

$0=-\frac{\partial p}{\partial x}+\frac{\partial}{\partial y}\left(e^{\alpha y} \frac{\partial u}{\partial y}\right)$

$0=-\frac{\partial p}{\partial y}$

Eq. (20) reveals that $p$ is function of $y$ alone.

The set of eqs. (19-20), subject to the conditions (10) are solved exactly for $u$, we have.

$u=\frac{(\partial p / \partial x)}{\alpha^{2}}\left(\alpha y e^{\alpha y}-e^{\alpha y}\right)+\frac{A}{\alpha(\partial p / \partial x)} e^{\alpha y}+\frac{B}{(\partial p / \partial x)}$,

The corresponding stream function is

$\psi(y)=\frac{(\partial p / \partial x)}{\alpha^{3}}(\alpha y-2) e^{\alpha y}+\frac{A}{(\partial p / \partial x) \alpha^{2}} e^{\alpha y}+\frac{B y}{(\partial p / \partial x)}+C$,

We can find that

$\frac{\partial p}{\partial x}=\frac{\alpha^{3} Q}{\left(\alpha h_{1}-2\right) e^{\alpha h_{1}}-\left(\alpha h_{2}-2\right) e^{\alpha h_{2}}+A \alpha\left(e^{\alpha h_{1}}-e^{\alpha h_{2}}\right)+B \alpha^{2}\left(h_{1}-h_{2}\right)}$. 
Where

$$
\begin{array}{ll}
A=\frac{\left[e^{\alpha h_{2}}\left(\alpha h_{2}-1\right)-e^{\alpha h_{1}}\left(\alpha h_{1}-1\right)\right]}{\alpha\left(e^{\alpha h_{1}}-e^{\alpha h_{2}}\right)}, & B=\frac{e^{\alpha\left(h_{2}+h_{1}\right)}\left(h_{2}-h_{1}\right)}{\alpha\left(e^{\alpha h_{2}}-e^{\alpha h_{1}}\right)}, \\
C & =\frac{2(\partial p / \partial x)-A \alpha}{\alpha^{3}} .
\end{array}
$$

The non-dimensional expressions for the average pressure rise $\Delta p_{\lambda}$,

$$
\Delta p_{\lambda}=\int_{0}^{1} \frac{\partial p}{\partial x} d x
$$

\section{Result and Discussion}

In prescribe to discuss the pumping characteristics of the expression (24) is not integral analytically therefore numerical integration is used to calculated the integrals using Mathematica and the results are presented graphically. Our objective to see the salient features of various physical parameters e.g., non-uniform parameter $k$, phase difference $\phi$, variable viscosity $\alpha$ and amplitudes ( $a$ and $b$ ) on pressure rise $\Delta p_{\lambda}$.

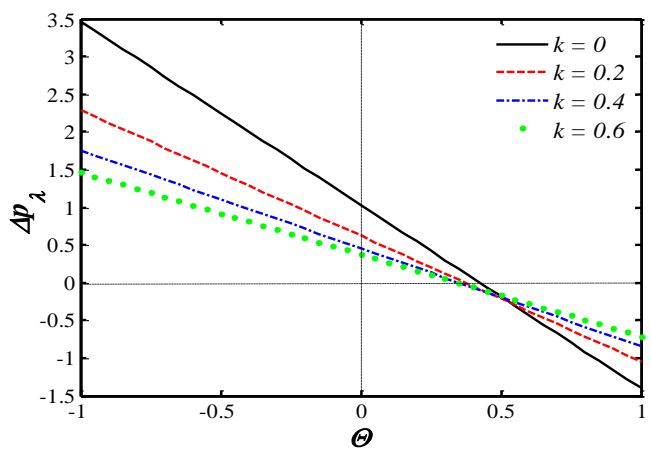

Fig. 2: The pressure rise versus flow rate for $a=0.5 ; b=0.4$; $\phi=\pi / 2$ and $\alpha=0.1$.

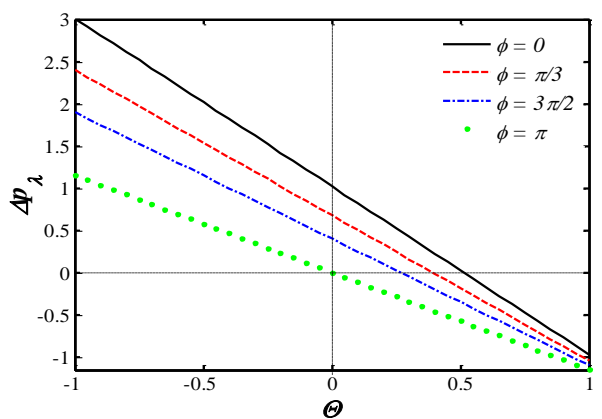

Fig. 3. The pressure rise versus flow rate for $a=0.5 ; b=0.4$; $k=0.2$ and $\alpha=0.1$.

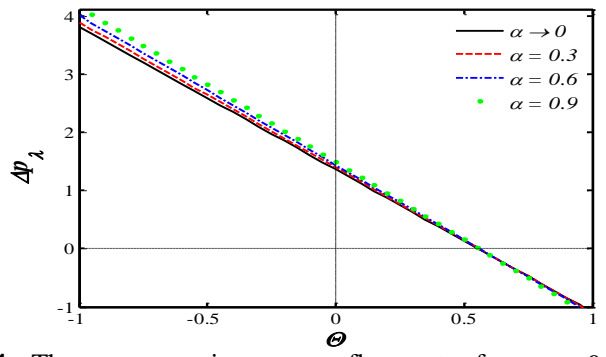

Fig. 4. The pressure rise versus flow rate for $a=0.6 ; b=0.7$; $k=0.1 ;$ and $\phi=\pi / 2$.

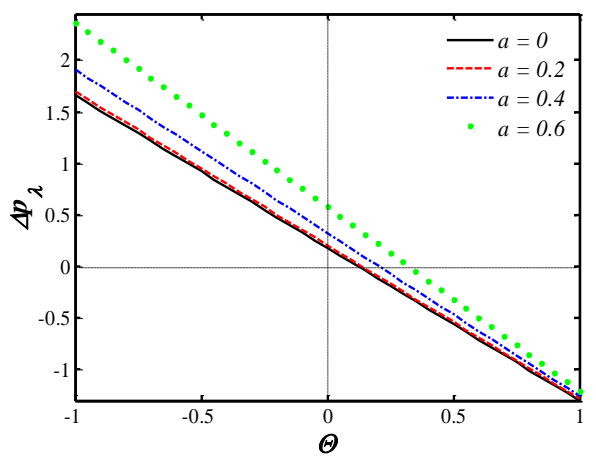

Fig. 5. The pressure rise versus flow rate for $b=0.4 ; k=0.1$ $\phi=\pi / 2$ and $\alpha=0.01$.

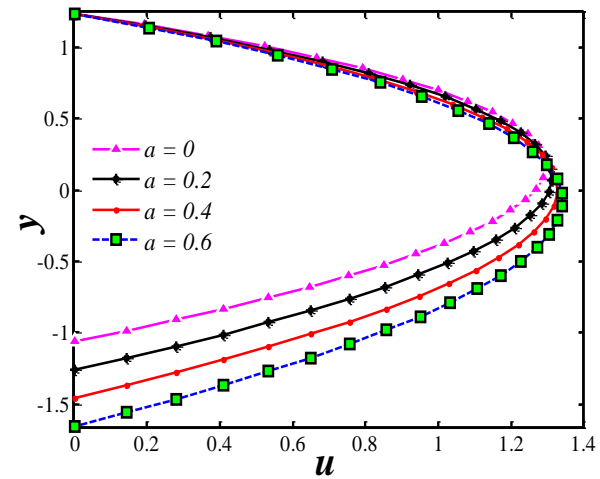

Fig. 6. The effect of $a$ on velocity profiles $b=0.3 ; k=0.2$ $\Theta=1.8 ; \phi=\pi / 3 ; \alpha=0.2$, at $x=0.3$ and $t=0.2$.

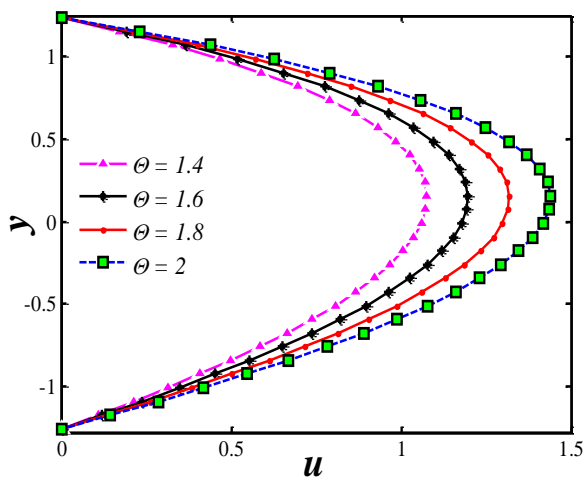

Fig. 7. The effect of $\Theta$ on velocity profiles at $a=0.2 ; b=0.3$; $k=0.2 ; \phi=\pi / 3 ; \alpha=0.3, x=0.3$ and $t=0.2$.

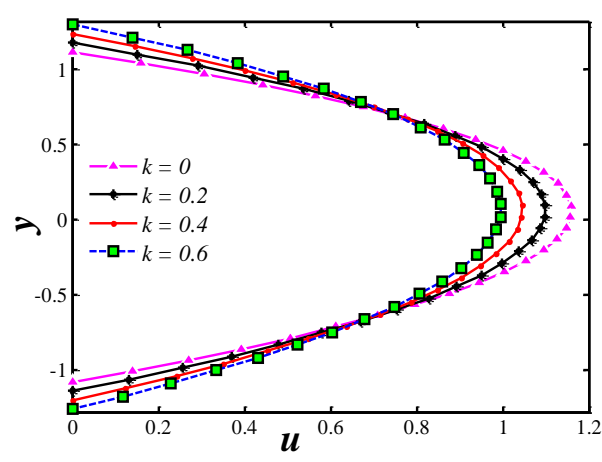

Fig. 8. The effect of $k$ on velocity profiles at $\Theta=1.5 ; \phi=\pi / 2 ; \alpha=0.1, x=0.3$ and $t=0.2$. 


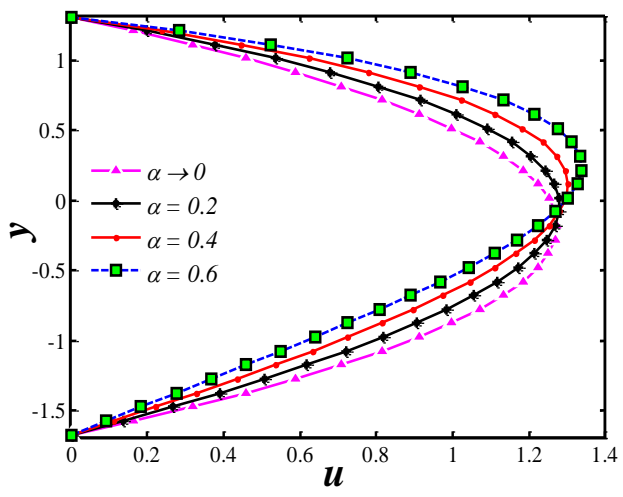

Fig. 9. The effect of $\alpha$ on velocity profiles at $a=0.6 ; b=0.4$; $k=0.25 ; \phi=\pi / 3 ; \Theta=1.7, x=0.3$ and $t=0.2$.

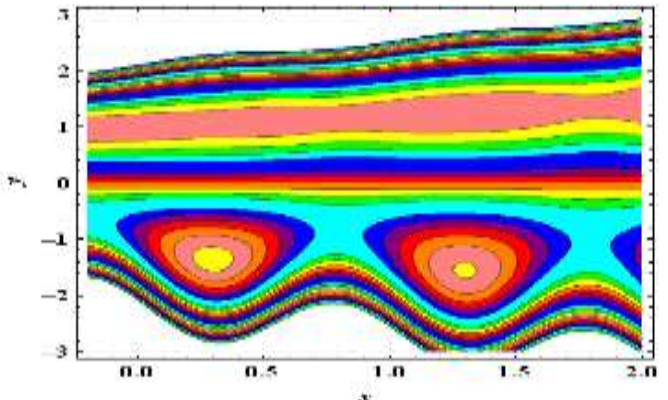

(a)

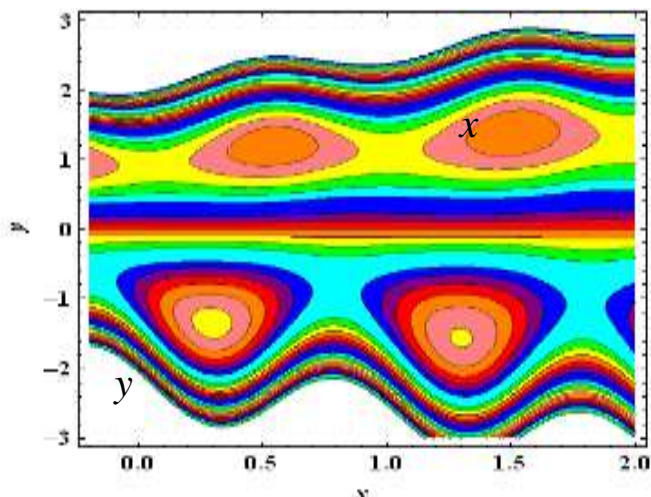

(b)

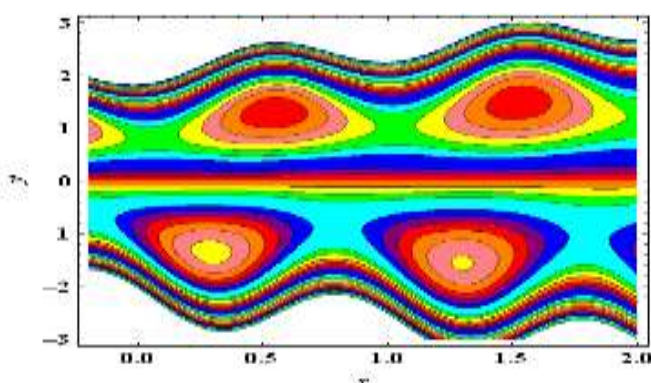

(c)

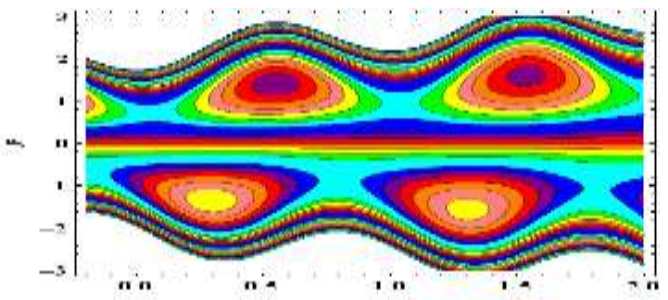

(d)

Fig. 10: Plot showing streamlines for four different parameter $b$ values $b=0$ (panel (a)), $b=0.1$ (panel (b)), $b=0.2$ (panel (c)) and $b=0.3$. (panel (d)). The other parameters chosen are $a=0.3, m=0.2, \phi=\pi / 2, \Theta=1.5, \alpha=0.1, t=0.3$.

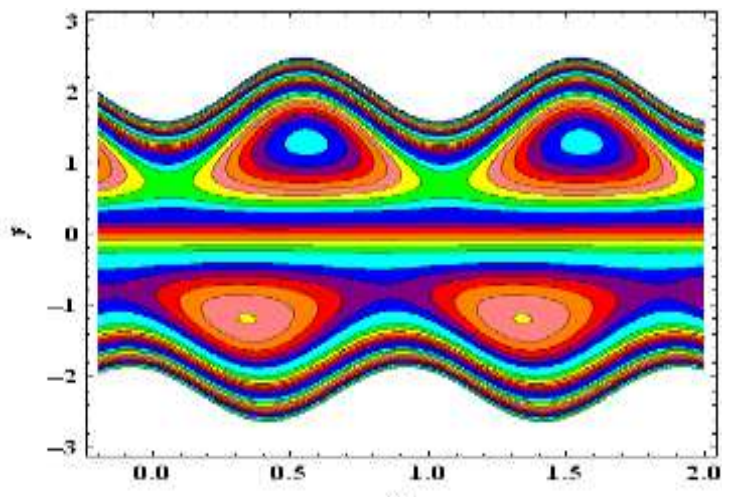

(a)

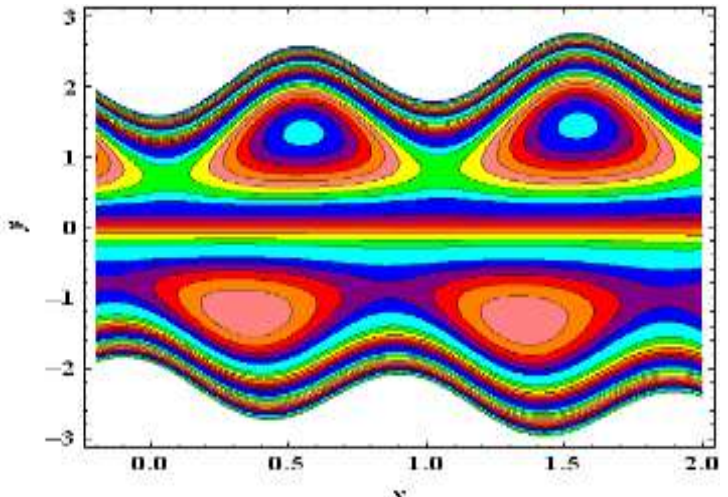

(b)

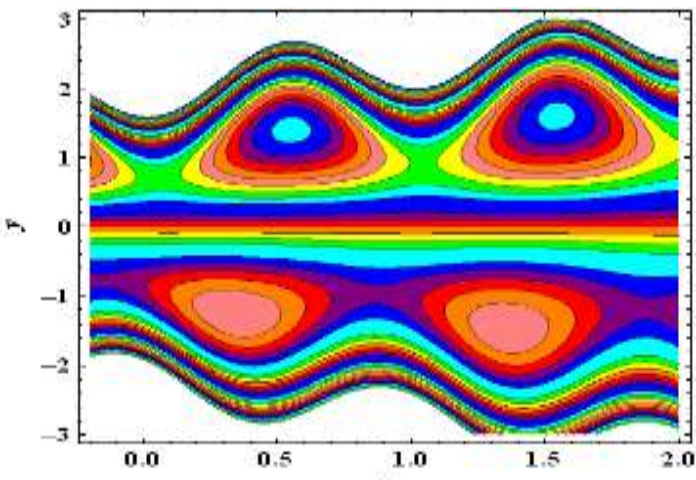

(c)

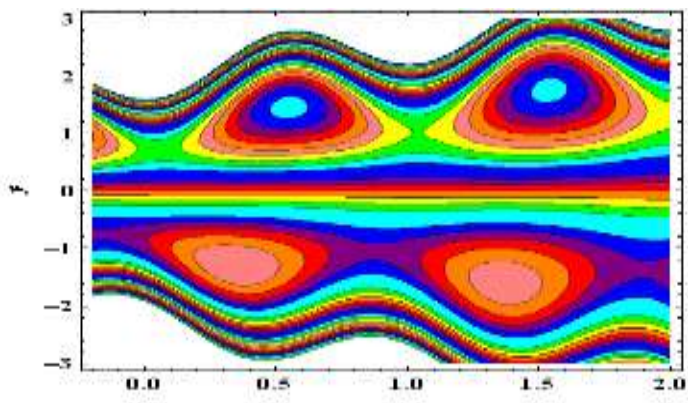

(d)

Fig. 11. Plot showing streamlines for four different parameter $k$ values $k=0$ (panel (a)), $k=0.1$ (panel (b)), $k=0.2$ (panel (c)) and $k=0.3$. (panel (d)). The other parameters chosen are $a=0.2, b=0.3, \phi=\pi / 3, \alpha=0.2, \Theta=1.7, t=0.3$. 


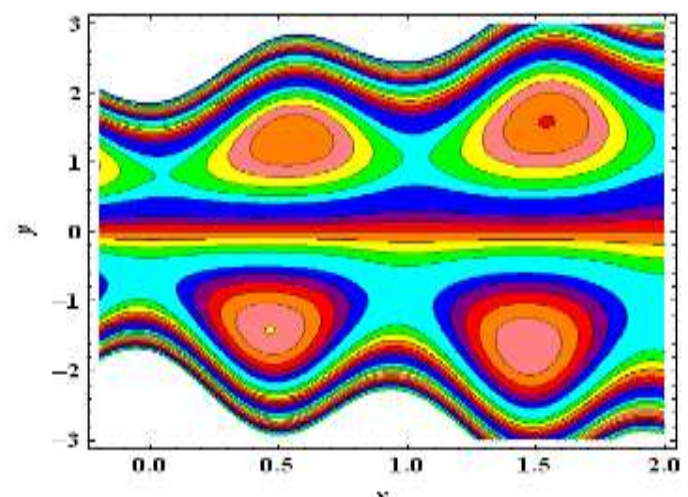

(a)

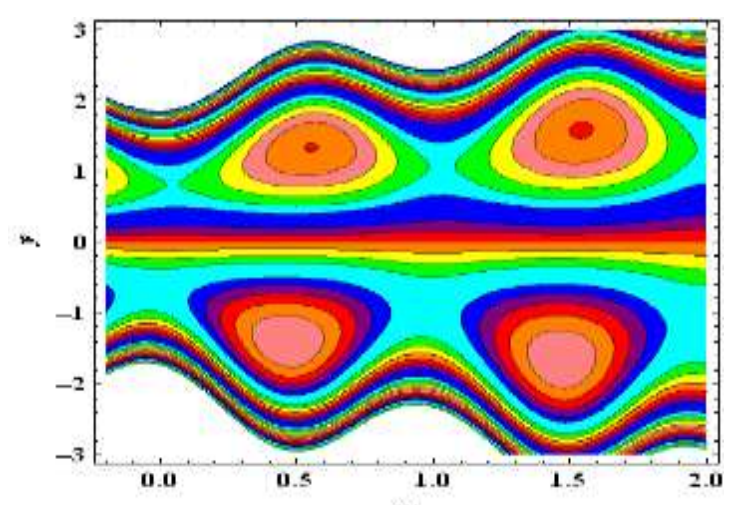

(b)

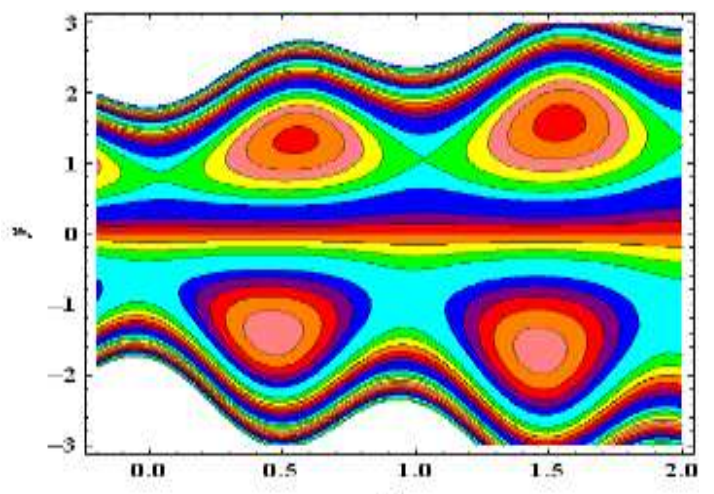

(c)

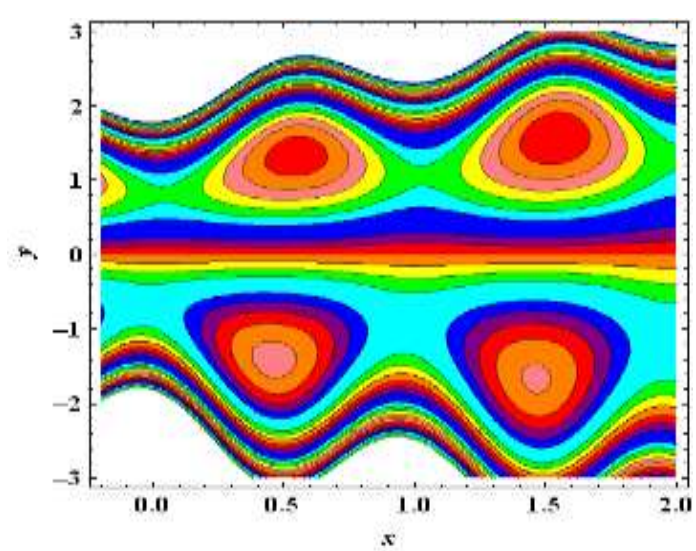

(d)

Fig. 12. Plot showing streamlines for four different parameter $\alpha$ values $\alpha \rightarrow 0$ (panel (a)), $\alpha=0.01$ (panel (b)), $\alpha=0.1$ (panel (c)) and $\alpha=0.2$ (panel (d)). The other parameters chosen are $a=0.3, b=0.2, \phi=\pi / 6, k=0.25, \Theta=1.4, t=0.3$.
The average pressure rise $\Delta p_{\lambda}$ versus time-averaged mean flow rate $\Theta$ is plotted, in Figs. (2-5), which show a linear relation between them. There are four types of regions regarding pumping. The graph is secured so that the upper right hand quadrant (I) denotes the region of peristaltic pumping where $\Theta>0$ and $\Delta p>0$. Quadrant (II), where $\Delta p<0$ and $\Theta>0$, are designated as augmented flow. Quadrant (IV) such that $\Delta p>0$ and $\Theta<0$ is called retrograde or backward pumping and for $\Delta p=0$ is a free pumping region. Fig. 2 presents the variation of $\Delta p_{\lambda}$ versus $\Theta$ for different value of non-uniform parameter $k$. It is observed that the average pressure rise decreases with increase of $k$. Fig.3 shows the effects of $\phi$ on the average pressure rise $\Delta p_{\lambda}$. It is clear that an increase in $\phi$ results in a decrease of the peristaltic pumping rate, free pumping $(\Delta p=0)$ and the adverse pressure rise $(\Delta p>0)$. In Fig.4, it is seen that the maximum pressure rise happens at zero time-averaged flow rate for different values of viscosity parameter $\alpha$. In addition, the average pressure rise increases by increasing viscosity parameter $\alpha$ also average pressure rise increases as time-averaged flow rate decreases. Fig.5, have been plotted to see the influence of average pressure rise $\Delta p_{\lambda}$, against time-averaged mean flow rate for different values of amplitude of lower wall $a$. It is depicted that average pressure rise increases by increasing amplitude of lower wall $a$.

Figs. 6-9 are prepared for the velocity field versus $y$. It is noticed from Fig. 6 that the velocity profile is parabolic nature and it increases with an increase in dimensionless amplitude of lower wall $a$. Fig. 7 displays the effects of mean flow rate $(\Theta)$ on the axial velocity distribution. It is found that the axial velocity increases as $\Theta$ increases. The effect of the non - uniform parameter $k$ on $u$ is illustrated in Fig. 8. It is exposed that the axial velocity $u$ increases in near the tapered microchannel walls while the reverse situation is observed in the hub part of the trapped channel. The velocity distribution for increasing values of $\alpha$ is shown in Fig. 9. One of the important findings of the present study is that the maximum velocity does not occur in the middle of the tapered microchannel, but moves towards the upper wall with the increase in the variable viscosity difference between the walls.

The establishment of an internally circulating bolus of fluid by closed streamlines is called trapping and this trapped bolus is forced in front along with the peristaltic wave. The results of $a, k, \alpha$ and $\Theta$ on trapping can be seen through Figs 10-12. The effect of amplitude of upper wall $b$ on trapping is studied through Fig. 10. It is seen that the size of the trapping bolus increases by increasing $b$. The streamlines for different values $k$ are shown in Fig.11. It is evident from the figure that the size of the trapped bolus increases with an increase in $k$. To see the effects of viscosity parameter $\alpha$, Fig. 12 is plotted. It is observed that when we increase $\alpha$, the size of trapped bolus increases in the amplitude of upper part of the channel and reverse situation happens in amplitude of lower wall.

\section{Concluding Remarks}

We have theoretically analyzed the problem of peristaltic transport of a viscoelastic fluid in the tapered microchannel with variable viscosity. The governing two dimensional equations have been modeled and then simplified using long wavelength and low Reynolds number approximations. Closed form expression of stream function, axial velocity and pressure gradient are developed. The results are discussed through graphs. We conclude the following observations:

$>$ The free pumping flux for a fluid with variable viscosity is greater as equated to fluid with constant viscosity. Further- 
more, peristalsis has to work versus a greater pressure for a fluid with constant viscosity when compared with that fluid of variable viscosity.

$>$ It is found that the pressure rise increases as the variable viscosity parameter and amplitude of lower wall parameter increases and it decreases as the non-uniform parameter and phase difference.

$>$ It is interesting to note that the fluid axial velocity profile increases as its viscosity increases. This can be assigned to the fact that the fluid has becomes lighter and then flow quicker.

$>$ It is concluded that when we increase $\alpha$, the size of trapped bolus decreases in the amplitude of lower part of the channel but it is reversed situation happens in amplitude of upper wall.

\section{References}

[1] Pozrikidis CA. A study of peristaltic flow. J Fluid Mech 1987; 180:515-27.

[2] Eytan Osnat, Elad David. Analysis of intrauterine fluid motion by uterine contraction. Bull Math Biol 1999;61:221-38.

[3] M. Kothandapani, J. Prakash, V. Pushparaj, Nonlinear peristaltic motion of a Johnson-Segalman fluid in a tapered asymmetric channel, Alexandria Engineering Journal, 55(1), (2016) 645-651.

[4] Haroun MH. Non linear peristaltic transport flow of a fourth grade fluid in an inclined asymmetric channel. Comput Sci 2007;39:32433.

[5] Herwig H, Wickern G. The effect of variable properties on laminar boundary layer flow. Wrme Stoffubert 1986;20:45-57.

[6] Elshehawey EF, Eladabe NT, Elghazy EM, Ebaid A. Peristaltic transport in an asymmetric channel through a porous medium. Applied Mathematics and Computation 2006; 182:140-150.

[7] M Kothandapani, J Prakash and V Pushparaj, Analysis of heat and mass transfer on MHD peristaltic flow through a tapered asymmetric channel, Journal of fluids, 2015, Article ID 561263

[8] M. Kothandapani, J. Prakash, S. Srinivas, Peristaltic transport of a MHD Carreau fluid in a tapered asymmetric channel with permeable walls, International Journal of Biomathematics, 08, 1550054 (2015).

[9] Misra JC, Pandey SK. A mathematical model for oesophageal swallowing of a food-bolus. Applied Mathematics and Computation 2001; 33:997-1009.

[10] Hariharan P, Seshadri V, Banerjee RK. Peristaltic transport of nonNewtonian fluid in a diverging tube with different wave forms. Mathematical and Computer Modelling 2008; 48:998-1017.

[11] M. Kothandapani, J. Prakash, Influence of thermal radiation and magnetic field on peristaltic transport of a Newtonian nanofluid in a tapered asymmetric porous channel, Journal of nanofluid, vol.05 (3), (2016) 363-374

[12] Eytan.O, and Elad. D., Analysis of Intra - Uterine fluid motion induced by uterine contractions,Bull. Math. Bio. vol.61 (1999), pp.221-238.

[13] Eytan.O, Jaffa. A.J., and Elad.D, Peristaltic flow in a tapered channel: application to embryo transport within the uterine cavity, Med. Engng. Phys., vol.23 (2001), pp. 473-482.

[14] Mishra.M. and Ramachandra Rao.A., Peristaltic transport of a Newtonian fluid in an asymmetric channel, Z. Angew. Math. Phys. (ZAMP), vol.54 (2003), pp.532-550.

[15] Hayat, T., Ali, N., 2007. Peristaltic motion of Carreau fluid in an asymmetric channel. Applied Mathematics and Computation 193, $535-552$

[16] K. Vajravelu, S. Sreenadh , K. Rajanikanth, Changhoon Lee, Peristaltic transport of a Williamson fluid in asymmetric channels with permeable walls , Nonlinear Analysis: Real World Applications 13 (2012) 2804-2822.

[17] Haynes, H.R., 1960. Physical basis of the dependence of blood viscosity on tube radius. American Journal of Physiology 198, 1193 1200 .

[18] Bugliarello, G., Sevilla, J., 1970. Velocity distribution and other characteristics of steady and pulsatile blood flow in fine glass tubes. Biorheology 7, 85-107.

[19] Wiedeman, M.P., 1963. Dimensions of blood vessels from distributing artery to collecting vein. Circulation Research 12, 375-381.

[20] Lee, J.S., Fung, Y.C., 1971. Flow in non-uniform small blood vessels. Microvascular Research 3, 272-279.
[21] L.M. Srivastava, V.P. Srivastava, Peristaltic transport of a nonNewtonian fluid (application to the vas deferens at small intestine), Annals of Biomedical Eng. 13 (1985) 137-153.

[22] Tasawar Hayat, Nasir Ali, Effect of variable viscosity on the peristaltic transport of a Newtonian fluid in an asymmetric channel, Applied Mathematical Modelling 32 (2008) 761-774.

[23] Ali N, Hussain Q, Hayat T, Asghar S. Slip effects on the peristaltic transport of MHD fluid with variable viscosity. Physics Letters A 2008; 372:1477-1489.

[24] Osnat Eytan, Ariel J. Jaffa, David Elad, 2001. Peristaltic flow in a tapered channel: application to embryo transport within the uterine cavity. Medical Engineering \& Physics 23, 473 - 482.

[25] Kikuchi Y. Effect of Leukocytes and platelets on blood flow through a parallel array of microchannels: micro-and Macroflow relation and rheological measures of leukocytes and platelate acivities. Microvasc Res 1995, 50, 288-300. 